\title{
Topical application of marine briarane-type diterpenes effectively inhibits 12-0- tetradecanoylphorbol-13-acetate-induced inflammation and dermatitis in murine skin
}

Wen-Chi Wei ${ }^{1,2}$, Sheng-Yen Lin ${ }^{1,3}$, Yi-Jyun Chen ${ }^{1,4}$, Chih-Chun Wen ${ }^{1}$, Chiung-Yao Huang ${ }^{2}$, Arulselvan Palanisamy ${ }^{1}$, Ning-Sun Yang ${ }^{1,4,5^{*}}$ and Jyh-Horng Sheu ${ }^{4,6^{*}}$

\begin{abstract}
Background: Skin is the largest organ in the body, and is directly exposed to extrinsic assaults. As such, the skin plays a central role in host defense and the cutaneous immune system is able to elicit specific local inflammatory and systemic immune responses against harmful stimuli. 12-O-tetradecanoylphorbol-13-acetate (TPA) can stimulate acute and chronic inflammation and tumor promotion in skin. TPA-induced dermatitis is thus a useful in vivo pharmacological platform for drug discovery. In this study, the inhibitory effect of briarane-type diterpenes (BrDs) from marine coral Briareum excavatum on TPA-induced dermatitis and dendritic cell (DC) function was explored.

Methods: Evans blue dye exudation was used to determine vascular permeability. H\&E-stained skin section was used to determine the formation of edema in mouse abdominal skin. We also used immunohistochemistry staining and western blot assays to evaluate the activation of specific inflammation makers and key mediators of signaling pathway in the mouse skin. Furthermore, mouse bone marrow DCs were used to determine the relationship between the chemical structure of BrDs and their regulation of DC function.

Results: BrD1 remarkably suppressed TPA-induced vascular permeability and edema in skin. At the biochemical level, BrD1 inhibited TPA-induced expression of cyclooxygenase-2, inducible nitric oxide synthase and matrix metalloproteinase-9, the key indicators of cutaneous inflammation. This inhibition was apparently mediated by interference with the Akt/NF- $\kappa$ B-mediated signaling network. BrD1 also inhibited TNF- $\alpha$ and IL- 6 expression in LPSstimulated BMDCs. The 8, 17-epoxide of BrDs played a crucial role in the inhibition of IL-6 expression, and replacement of the C-12 hydroxyl group with longer esters in BrDs gradually decreased this inhibitory activity.
\end{abstract}

Conclusions: Our results suggest that BrDs warrant further investigation as natural immunomodulatory agents for control of inflammatory skin diseases.

Keywords: Briarane-type diterpene, TPA-induced mouse dermatitis, Dendritic cells, Vascular permeability, Edema

\section{Background}

Skin is the largest organ in the body. As the primary interface between the body and environment, it serves as the first line of defense against microbial pathogens as well as physical and chemical stress or insults $[1,2]$.

\footnotetext{
* Correspondence: nsyang@gate.sinica.edu.tw; sheu@mail.nsysu.edu.tw ${ }^{1}$ Agricultural Biotechnology Research Center, Academia Sinica, Taiwan ${ }^{4}$ Department of Life Science, National Central University, Taoyuan County, Taiwan

Full list of author information is available at the end of the article
}

The skin does not only serve as a physical and a chemical barrier, but is also an immune-competent organ that elicits effective innate and adaptive immune responses to protect the human body. The cutaneous immune system maintains a balance between restricting excessive inflammation following tissue damage or injury and preserving the ability to rapidly respond to pathogen infection [3]. It is clear that inadequate or misdirected immune response is involved in the pathogenesis of a variety of acquired inflammatory skin disorders [1].

\section{Biomed Central}


Therefore, systematic investigation of the mechanisms of action of immunomodulatory agents on the skin's immune system is necessary for the development of therapies for skin disorders.

Acute inflammation is the initial immune response to harmful stimuli. Acute inflammation in the skin often involves an increase in the vascular permeability of skin tissues, resulting in an accumulation of fluid at the inflamed site (edema). The release of mediator molecules such as nitric oxide and prostaglandins also elicits vascular permeability, thus permitting the efficient migration of leukocytes, mainly neutrophils, to the inflamed tissue site. Matrix metalloproteinase-9 (MMP9) has been reported to be a crucial player in such neutrophil migration by degrading some major cellular components of the epidermis and dermis [4]. In addition, it is well-known that secretions of cytokines such as TNF- $\alpha$, IL- $1 \alpha$ and IL- 6 by keratinocytes or antigenspecific cells can play a key role in mediating the cutaneous inflammatory response $[2,5,6]$. These mediators were employed as indicators of skin inflammation in this study.

Several novel approaches have been explored to manage risk factors for skin cancers, tissue damage from UVB exposure, and inflammatory skin disorders [7]. Several phytochemicals and tissue extracts from medicinal plants have been reported to confer immunostimulatory activities and have potential clinical applications $[8,9]$. Our laboratories previously reported that a small phytochemical from Lithospermum erythrorhizon (shikonin) can inhibit the transcriptional activation of human TNF- $\alpha$ promoter in vivo in mouse skin [10]. We also showed that caffeic acid suppresses UVB radiation-induced expression of IL-10 and activation of MAPKs in mouse skin tissues [11]. More recently, we demonstrated that ferulic acid, a phenolic phytochemical, can effectively inhibit UVB-induced matrix metalloproteinases in mouse skin via a posttranslational mechanism [7].

Natural products from plants and terrestrial microorganisms have traditionally provided good sources of lead compounds/agents for human medicines. However, due to the biological diversity of the marine environment and the discovery of marine compounds with certain unique structures and pharmacological activities, compounds from marine organisms are expected to be a major source of lead compounds for future generations of pharmaceuticals [12]. A spectrum of different novel marine compounds have been identified, and their bioactivities evaluated for potential pharmaceutical application [13]. Among these compounds, we isolated and identified a group of briarane-type diterpenes (BrDs) from Briareum excavatum, a Formosan gorgonian coral $[14,15]$.
In this study, we selected a group of specific BrDs from our chemical library of marine origin for systematic study and showed that BrD1 (excavatolide B) can drastically inhibit 12-O-tetradecanoylphorbol-13-acetate (TPA)-induced acute inflammation in murine skins, as determined by its effect on vascular permeability, edema and key inflammatory mediators. We further determined a structure-activity relationship (SAR) of selected BrDs in terms of their regulation of cytokine expression. The possible molecular mechanisms for the mode of action of these BrDs were also studied.

\section{Methods}

\section{Reagents}

Recombinant cytokines mIL-4 and mGM-CSF were purchased from PeproTech (Rocky Hill, NJ). TPA (12-Otetradecanoyl-13-phorbol-acetate), Evans blue dye, $N, N$ dimethyl-formamide and LPS (Escherichia coli 055:B5) were purchased from Sigma-Aldrich (St. Louis, MO). BrDs were prepared from marine soft coral (Briareum excavatum) as previously described [14].

\section{Mice}

Female C57BL/6JNarl mice (5-6 weeks old) were purchased from the National Laboratory Animal Breeding and Research Center, Taipei, Taiwan. All mice were maintained in a laminar airflow cabinet in a specific pathogen free (SPF) animal room kept at $24 \pm 2^{\circ} \mathrm{C}$ and $40-70 \%$ humidity with a $12 \mathrm{~h}$ light/dark cycle under SPF conditions. All facilities were approved by the Institutional Animal Care and Utilization Committee of Academia Sinica, and animal experiments were all conducted according to institutional guidelines.

\section{Preparation of 12-acyloxyl analogues of BrD1}

An appropriate acyl chloride $(0.02 \mathrm{mmol})$ was added to a solution of BrD1 (excavatolide B) $(10 \mathrm{mg})$ in $5 \mathrm{ml}$ of pyridine; the mixture was allowed to stand overnight at room temperature. Four milliliters of water was added to the reaction mixture followed by extraction with EtOAc $(5 \mathrm{ml} \times 3)$. The EtOAc layers were combined, dried over anhydrous $\mathrm{MgSO}_{4}$ and evaporated. The afforded residue was purified by column chromatography on silica gel using EtOAc/hexane (1:8) as eluent to yield analogues of BrD1 (BrD1-5C, BrD1-6C, BrD1-7C and $\mathrm{BrD} 1-10 \mathrm{C})$. Yields varied from $61.6 \%$ to $78.3 \%$.

\section{Measurement of vascular permeability}

TPA-induced vascular permeability assay was modified and performed as previously described [16]. Shaved abdominal skins of female C57BL/6JNarl mice were topically treated with vehicle (acetone, $200 \mu \mathrm{l} / \mathrm{site}$ ) or TPA (10 nmol in $200 \mu \mathrm{l}$ acetone/site) for $6 \mathrm{~h}$ or treated with TPA for $10 \mathrm{~min}$ and subsequently treated for $6 \mathrm{~h}$ 
with the indicated concentrations of BrD1. Abdominal skins of untreated mice were used as the control group. One percent Evans blue dye $(100 \mu \mathrm{l})$ was injected into mouse tail veins. After $20 \mathrm{~min}$, anatomical appearances of mouse abdomens from the various treatments were photographically recorded. Abdominal skins representative of each test group were removed, turned over and photographed. Evans blue dye extravasated into the skins was extracted by incubation of the skin samples in $99 \% \mathrm{~N}, \mathrm{~N}$-dimethyl-formamide overnight at $60^{\circ} \mathrm{C}$ and optical density was measured at $620 \mathrm{~nm}$.

Generation of mouse bone marrow-derived dendritic cells For mouse bone marrow-derived DCs (BMDCs), five to six-week-old female C57BL/6JNarl mice were purchased from the National Laboratory Animal Center, Taiwan and kept under SPF conditions. BMDCs were generated from bone marrow cells of C57BL/6 mice as described previously [17]. In brief, bone marrow was isolated from femurs and tibiae which were then flushed with RPMI1640 medium using a syringe with a $0.45-\mathrm{mm}$ needle on Day 0. Red blood cells in suspension were lysed for $5 \mathrm{~min}$ with ACK lysing buffer $\left(150 \mathrm{mM} \mathrm{NH}_{4} \mathrm{Cl}, 1.0 \mathrm{mM}\right.$ $\mathrm{KHCO}_{3}, 0.1 \mathrm{mM}$ EDTA). Bone marrow cells were suspended at a density of $1 \times 10^{7}$ cells $/ 30 \mathrm{ml}$ in RPMI-1640 containing 10\% FBS, $2 \mathrm{mM} \mathrm{L}$-glutamine, $1 \%$ of nonessential amino acids, $100 \mathrm{U} / \mathrm{mL}$ penicillin and $100 \mu \mathrm{g} / \mathrm{mL}$ streptomycin supplemented with $20 \mathrm{ng} / \mathrm{mL}$ of mGMCSF in $15-\mathrm{cm}$ dishes at $37^{\circ} \mathrm{C}$ with $5 \% \mathrm{CO}_{2}$. On day 2 , two-thirds of the medium was removed and $30 \mathrm{~mL}$ fresh medium with mGM-CSF was added to the cells. On day 5 , culture plates were gently swirled and the floating and loosely adherent cells were discarded. Aliquots of $75 \%$ culture media were replenished with $20 \mathrm{ng} / \mathrm{mL} \mathrm{mGM}$ CSF and $20 \mathrm{ng} / \mathrm{mL}$ mIL-4. On day 7, mouse BMDCs (95\% pure CD11b and MHCII) were harvested and incubated in RPMI 1640 medium containing $1 \mathrm{mM}$ sodium pyruvate, $0.1 \mathrm{mM}$ nonessential amino acids, $100 \mathrm{U} / \mathrm{ml}$ penicillin, $100 \mu \mathrm{g} / \mathrm{ml}$ streptomycin, and supplemented with $10 \%$ fetal bovine serum (Invitrogen, Carlsbad, CA) for $24 \mathrm{~h}$ at $37^{\circ} \mathrm{C}$ with or without test chemicals in the presence or absence of LPS (100 ng/ml).

\section{Measurement of pro-inflammatory cytokines}

BMDCs were treated with or without test chemicals in the presence or absence of LPS $(100 \mathrm{ng} / \mathrm{ml})$ for $24 \mathrm{~h}$ at $37^{\circ} \mathrm{C}$. Aliquots of supernatants from $\mathrm{DC}$ cultures were assayed for IL- 6 and TNF- $\alpha$ using commercial ELISA kits (R\&D Systems, Minneapolis, MN) following the manufacturer's recommendations.

\section{Primer design and RT-PCR}

YES: Yesp Shaven abdominal skins of female C57BL/ 6JNarl mice were untreated or topically treated with vehicle (acetone, $200 \mathrm{ml} / \mathrm{site}$ ) or TPA (10 nmol in 200 $\mathrm{ml}$ acetone/site) for $6 \mathrm{~h}$ or treated first with TPA for 10 min and then with the indicated concentrations of BrD1 for $6 \mathrm{~h}$. Total RNA was extracted from treated abdominal skins of female mice using TRIzol reagent (Invitrogen Corp., Carlsbad, CA). RT-PCR reactions using AccessQuick RT-PCR system (Promega, Madison, WI) were carried out as described previously [7]. The primers contained the following sequences: mouse MMP-9 sense primer 5'-CTAGTGAGAGACTCTACACGGAG3', and anti-sense primer 5'-GAGCCACGACCATACAGATACTG-3'; mouse GAPDH sense primer 5'-CATCACTGCCACCCAGAAGACTGTGGA-3', and antisense primer 5'-TACTCCTTGGAGGCCATGTAGGCCATG-3'. Gel images were scanned and densitometry analysis of the captured image was performed using Gene Tools software (Syngene, Cambridge, UK).

\section{Histopathological analysis}

Mice were treated or not treated topically on their shaven abdomens with acetone (vehicle only) or TPA (10 $\mathrm{nmol}$ in $200 \mu \mathrm{l}$ acetone/site) for $6 \mathrm{~h}$ or treated first with TPA for $10 \mathrm{~min}$, then with the indicated concentrations of $\mathrm{BrD} 1$ for $6 \mathrm{~h}$. Mice were killed by cervical dislocation. Abdominal skin tissues were collected, fixed with formalin buffer and embedded in paraffin. Tissue sections (5 $\mathrm{mm}$ ) were cut and laid onto silanized glass slides and deparaffinized three times with xylene for $5 \mathrm{~min}$ each prior to rehydration using a graded alcohol bath. For hematoxyline and eosin staining, the sections were stained with hematoxyline and eosin staining. For immunohistochemical staining, the deparaffinized sections were boiled in $10 \mathrm{mM}$ citrate buffer ( $\mathrm{pH}$ 6.0) for $10 \mathrm{~min}$ for antigen retrieval and rinsed with PBS containing $0.05 \%$ Tween-20 buffer for $5 \mathrm{~min}$. The sections were treated with $3 \%$ hydrogen peroxide in methanol for $15 \mathrm{~min}$ to decrease non-specific binding. They were subsequently washed with blocking solution (PBS containing 1\% BSA) for $30 \mathrm{~min}$ and then PBST twice for 5 min. All slides were incubated first with $2 \%$ goat serum in blocking solution for $30 \mathrm{~min}$, then with a 1:200 dilution of polyclonal iNOS antibody (eBioscience, San Diego, CA) at room temperature for $1 \mathrm{~h}$. The slides were further developed using HPR EnVisionTM system (Dako, Glostrup, Denmark). Subsequently, peroxidasebinding sites were determined by staining with 3,3'-diaminobenzidine tetrahydrochloride (Dako). Eventually, Mayer's hematoxyline was used for counterstaining.

\section{Western blotting analysis}

Shaven abdominal skins of female C57BL/6JNarl mice were not treated or topically treated with vehicle (acetone, $200 \mu \mathrm{l} / \mathrm{site})$ or TPA (10 nmol in $200 \mu \mathrm{l}$ acetone/site) at indicated time points or treated first with TPA for $10 \mathrm{~min}$, 
then with the indicated concentrations of BrD1 at the indicated time points. Abdominal skin samples were collected, homogenated and lysed to prepare total proteins. Protein samples were subsequently resolved by SDS-PAGE using a gradient gel. The resolved proteins were transferred to a PVDF Immobilon-P membrane (Millipore, Bedford, CA.), and the membrane was blocked with $5 \%$ non-fat dry milk in PBST buffer (phosphate-buffered saline (PBS) containing $0.1 \%$ Tween 20 ) for 60 minutes at room temperature. The membranes were incubated overnight at $4^{\circ} \mathrm{C}$ with commercially available antibodies (1:1000 dilutions). Loading of equal amounts of protein was assessed using mouse $\beta$-actin. The blots were rinsed three times with PBST buffer for 5 minutes. Washed blots were incubated with HRP-conjugated secondary antibody (1:100,000 dilution), then washed again three times with PBST buffer. The transferred proteins were visualized with an enhanced chemiluminescence (ECL) detection kit (Amersham Pharmacia Biotech, Buckinghamshire, UK). Quantification of bands was performed using Image J software.

\section{Statistical analysis}

Statistical analyses were performed with GraphPad Prism software, version 5. Data are presented as mean \pm SD and statistical significance was determined by a oneway ANOVA followed by Tukey multiple comparison tests. Means were considered significantly different if the $\mathrm{P}$ value was less than 0.05 .

\section{Results}

\section{Excavatolide B (BrD1) effectively inhibits TPA-induced} vascular permeability in mouse skin

Acute inflammatory reactions are known to include changes in vascular permeability, edema and cellular infiltration [18]. In order to evaluate the anti-inflammatory effect of BrD1 on TPA-induced dermatitis in a murine model, we initially evaluated the possible inhibitory effect of BrD1 on TPA-induced vascular permeability. Abdomens of female C57BL/6 mice were treated topically with TPA $(10 \mathrm{nmol})$ or acetone (vehicle control) for $6 \mathrm{~h}$, or treated first with TPA for $10 \mathrm{~min}$ and then with the indicated concentrations of BrD1 for $6 \mathrm{~h}$. Mice were injected for $20 \mathrm{~min}$ via the tail vein with $100 \mu \mathrm{l}$ of $1 \%$ Evans blue. Consistent with previous reports, TPA strongly increased vascular permeability (Figure 1A, B and 1C). Topical application of BrD1 after TPA treatment significantly inhibited the TPA-induced vascular permeability. The level of inhibition was approximately $58 \%(0.5 \mathrm{mg} /$ site of BrD1) and $77 \%$ (1 mg/site of BrD1) (Figure 1C).

\section{BrD1 inhibits TPA-induced edema}

Since increased vascular permeability is one of factors contributing to the formation of edema, we next evaluated whether BrD1 inhibited formation of edema in TPA- induced inflammation by examining $\mathrm{H} \& \mathrm{E}$-stained longitudinal sections of skin samples. As seen in Figure 2, topical application of TPA markedly increased the skin thickness, especially in the hypodermal layer as compared with that of untreated or vehicle control groups. Treatment with BrD1 after TPA application substantially decreased the skin thickness, indicating BrD1 can effectively inhibit TPA-induced edema in mouse abdominal skin.

\section{BrD1 suppresses TPA-induced COX-2 and iNOS expression in mouse skin}

Cyclooxygenase-2 (COX-2) and inducible nitric oxide synthase (iNOS) are key mediators of various inflammation and immunity activities. A relatively high level of COX-2 and iNOS expression can be observed in skin during the acute phase of inflammation [19]. To examine whether BrD1 can also inhibit COX-2 and iNOS expression in TPA-inflamed skin, we applied BrD1 topically to TPA-treated skin for $6 \mathrm{~h}$, and then stained it immunohistochemically with anti-COX-2 and anti-iNOS antibodies. As seen in Figure 3A and 3B, TPA treatment stimulated COX-2 expression in the epidermal layer, and TPA treatment strongly stimulated iNOS production in the epidermal and dermal layers as compared with those of the untreated or vehicle control-treated mice. Furthermore, BrD1 treatment reduced the TPAinduced COX-2 expression in the epidermis (Figure 3A) and markedly reduced the TPA-induced iNOS expression in the epidermal and dermal layers of test mouse skin (Figure 3B).

\section{BrD1 inhibits TPA-induced MMP-9 expression in mouse skin}

Matrix metalloproteinases (MMPs) play a crucial role in many physiological and pathological processes through remodeling extracellular matrix tissues [20]. Matrix metalloproteinase-9 (MMP-9), a gelatinase which has a key role in inflammatory response, is activated in skin during tissue injury [21]. In order to evaluate the antiinflammatory effect of BrD1 on MMP-9 activation in TPA-inflamed skin, BrD1 was topically applied after TPA treatment for $24 \mathrm{~h}$. As seen in Figure 4A, TPA treatment vigorously stimulated MMP-9 protein expression as compared with untreated or vehicle control groups. On the other hand, BrD1 significantly inhibited TPA-induced MMP-9 protein expression in a dosedependent manner. Inhibition with BrD1 treatment (1 $\mathrm{mg} / \mathrm{site}$ /mouse) was up to a maximum $89 \%$. Inhibiton of TPA-induced MMP-9 expression by BrD1 treatment could also be readily observed at the mRNA level. As shown in Figure 4B, TPA strongly stimulated MMP-9 mRNA expression and BrD1 applied topically after TPA treatment also significantly inhibited MMP-9 mRNA expression in a dose-dependent manner. BrD1 treatment 
(A)

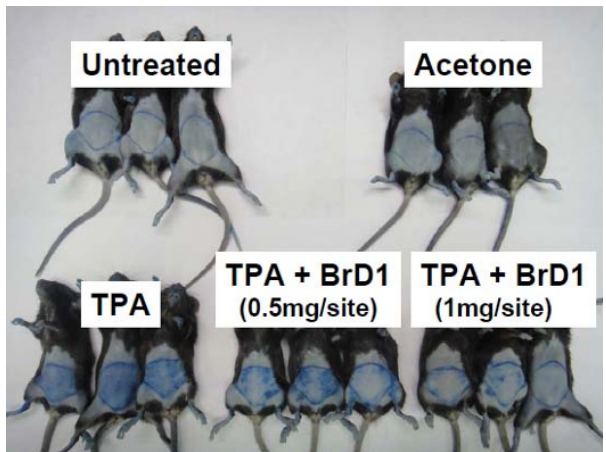

(B)

(C)
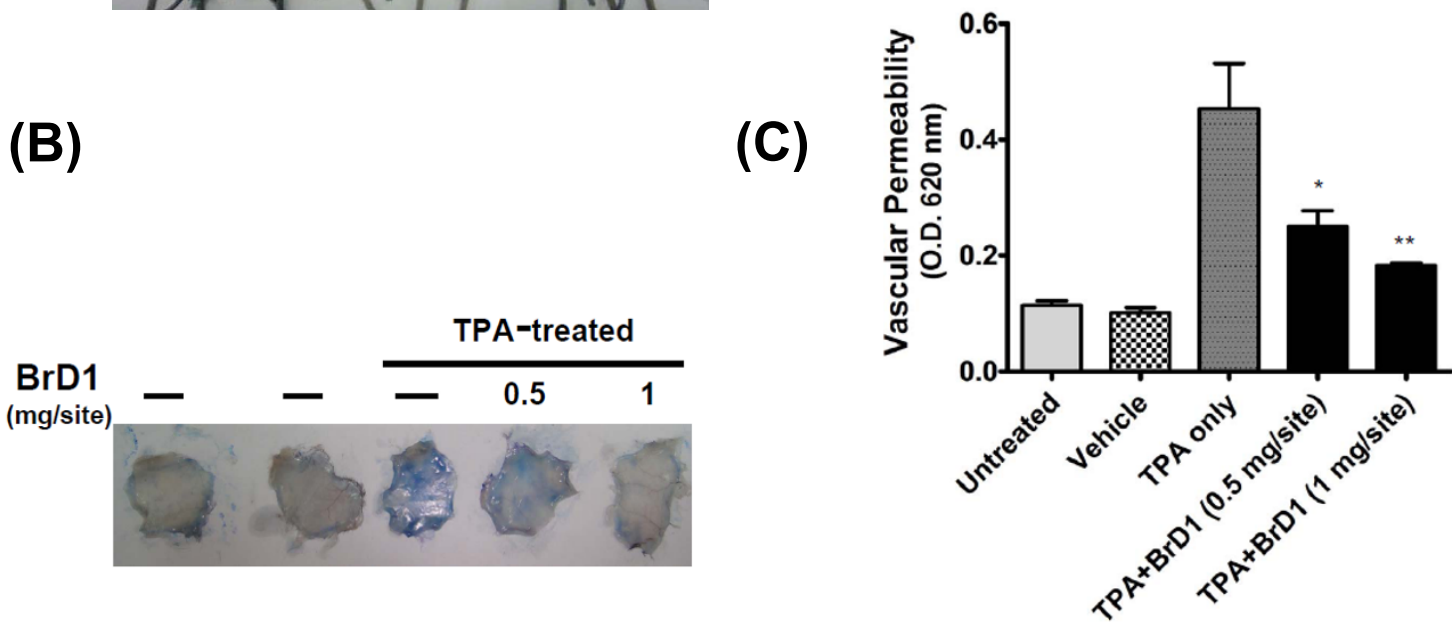

Figure 1 Briarane-type diterpene 1 (BrD1) inhibits TPA-induced vascular permeability in mouse skin. Abdominal skins of female C57BL/6 mice were treated topically with TPA $(10 \mathrm{nmol}$ ) or acetone (vehicle control) for $6 \mathrm{~h}$, or treated with TPA for 10 min and then treated for $6 \mathrm{~h}$ with the indicated concentrations of BrD1. One percent Evans blue dye (100 $\mu l)$ was injected into mouse tail veins for 20 min. (A) Photograph of mouse abdominal skins subjected to various treatments and vascular permeability test. (B) Photographs of the dermal (internal) sides of representative abdominal skins subjected to the above treatment and test. (C) Evans blue extravasation in test skins was determined by assay of optical density at $620 \mathrm{~nm}$. ${ }^{*}, P<0.05$, and ${ }^{*}, P<0.01$ versus LPS control. Data are representative of two independent experiments.

(1 mg/site/mouse) inhibited MMP-9 mRNA expression up to a maximum $82 \%$.

\section{BrD1 inhibits TPA-stimulated NF- $\kappa \mathrm{B}$ and Akt activation in mouse skin}

The roles of the Akt and Erk signaling pathways in inflammatory activities in mouse skin have been welldemonstrated. We therefore evaluated whether BrD1 could interfere with TPA-induced activation of Akt and Erk in inflamed skin. In order to assess the kinetics of Akt and Erk activation, abdominal skin was stimulated with TPA for 0.5 to $8 \mathrm{~h}$. A high level of phosphorylation of Akt and Erk was observed at $2 \mathrm{~h}$ after TPA treatment (Figure 5A). We therefore chose $2 \mathrm{~h}$ as the time point at which to assess the $\mathrm{BrD} 1$-associated signaling transduction activity in TPA-inflamed skin. The immuno-associated transcription factor NF- $\kappa \mathrm{B}$ is a key upstream mediator of iNOS and MMP-9 expressions, and is known to be involved in various cutaneous inflammatory responses. We thus determined the phosphorylation level of Akt, Erk, NF- $\kappa \mathrm{B}$ and I $\kappa \mathrm{B} \alpha$ in skins subjected to different treatments. TPA treatment strongly stimulated the phosphorylation of Akt, Erk, NF- $\kappa \mathrm{B}$ and $\mathrm{I} \kappa \mathrm{B} \alpha$ (Figure 5B). BrD1 seemed to inhibit TPA-induced Erk phosphorylation only slightly. However, BrD1 treatment effectively inhibited the phoshporylation of NF- $\kappa \mathrm{B}$, and also significantly inhibited the TPA-stimulated phosphorylation of Akt and $\mathrm{I} \kappa \mathrm{B} \alpha$. These results indicate that $\mathrm{BrD} 1$ can significantly inhibit TPA-stimulated NF- $\kappa$ B and Akt activation in test mouse skin.

BrD1 inhibits LPS-induced IL-6 and TNF- $\alpha$ expression in mouse bone marrow-derived dendritic cells

Several studies have reported that the secretion of specific cytokines, including IL- $1 \beta$, TNF- $\alpha$ and IL- 6 by keratinocytes and various immune cells is involved in cutaneous inflammation $[2,5,6]$. In order to investigate the anti-inflammatory effect of $\mathrm{BrD} 1$ on cytokine 


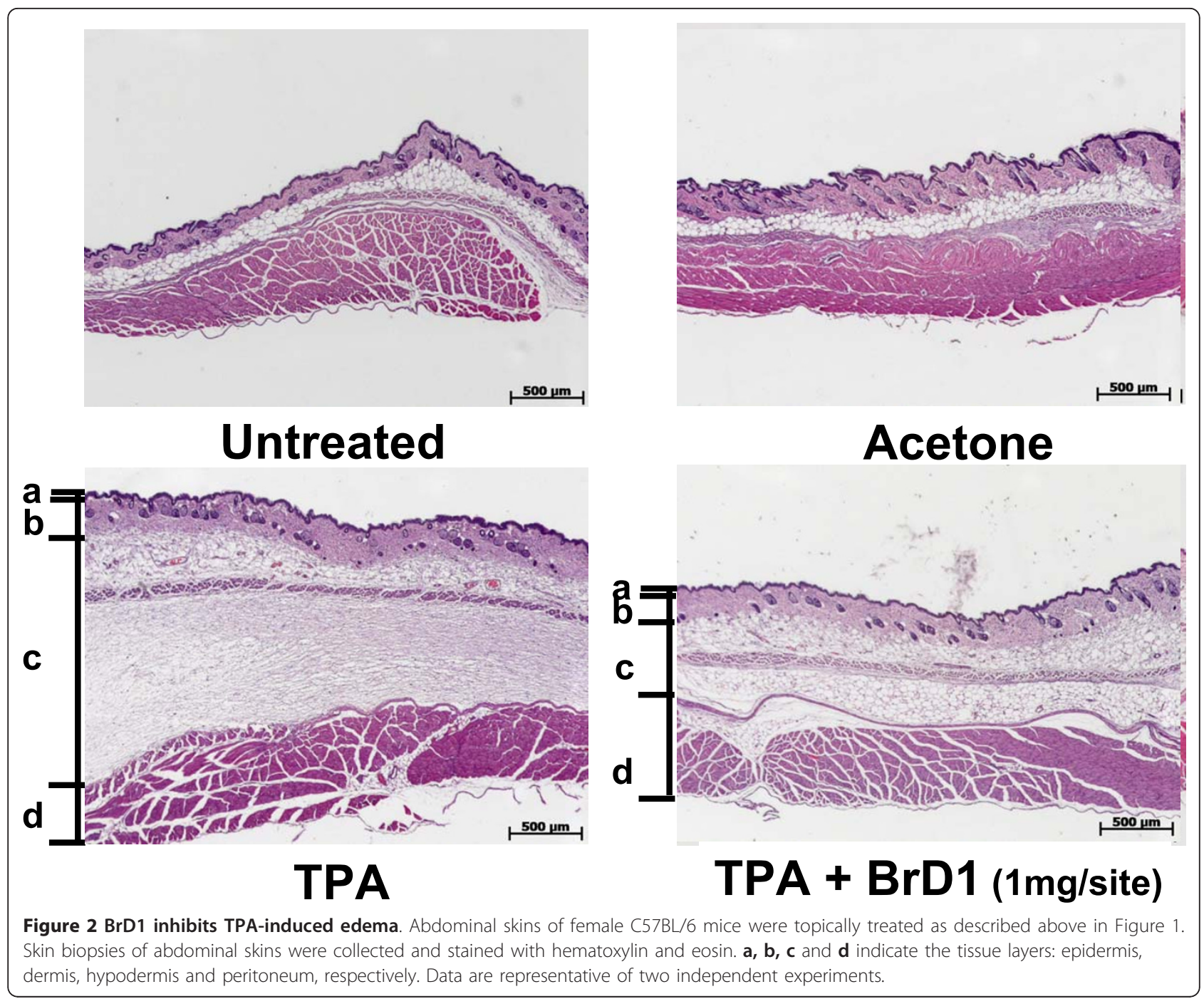

expression, mouse bone marrow-derived dendritic cells were used to evaluate the inhibitory effect of BrD1 on LPS-induced expressions of TNF- $\alpha$ and IL- 6 . In our time-course study for expression of TNF- $\alpha$ and IL- 6 , after LPS stimulation, a high expression of TNF- $\alpha$ and IL-6 was maintained at $24 \mathrm{~h}$ (Figure 6A). We therefore chose $24 \mathrm{~h}$ as the time point at which to assess the expression of TNF- $\alpha$ and IL-6. BrD1 treatment strongly inhibited IL- 6 expression in LPS-stimulated dendritic cells, and inhibited TNF- $\alpha$ expression to a much lesser extent (Figure 6B).

\section{Structure-activity relationship of BrDs that underlies inhibition of LPS-induced IL- 6 expression}

In order to explore the relationship between the chemical structures of the BrDs and their inhibition of inflammation, immature DCs were co-treated for $24 \mathrm{~h}$ with LPS and BrDs (BrD1-BrD8) at $20 \mu \mathrm{M}$. Initially, IL-6 was used as a target to evaluate the inhibitory activity of the
BrDs. As seen in Table 1 and Figure $7 \mathrm{~A}$, the BrDs exhibited a broad spectrum of inhibition in test mouse DCs. The range of inhibition was between 97.6\% (BrD1) and $18.7 \%$ (BrD8). Importantly, MTT cell viability assays showed that treatment with BrDs at the test concentration of $20 \mu \mathrm{M}$ for $24 \mathrm{~h}$ had no significant effect on the cytotoxicity of test DCs (data not shown). As shown in Table 1 and Figure 6, BrD1 to BrD6 with 8, 17-epoxides inhibited IL-6 expression more strongly than $\mathrm{BrD7}$ and $\mathrm{BrD} 8$ without 8,17 -epoxides indicating that the 8,17 epoxides of BrDs are crucial for the inhibition of LPSinduced IL- 6 expression by BrDs. In addition, BrD5 and BrD6 with $\alpha$-functional groups at $\mathrm{C}-12$ were found to inhibit IL-6 expression less vigorously than BrD1 to BrD4 without $\alpha$-functional groups at C-12, suggesting that the $\alpha$ position of the functional group at C-12 may have impaired the inhibitory capacity of these compounds (Table 1 and Figure 7A). Furthermore, replacement of the $\mathrm{C}-12$ hydroxyl group in $\mathrm{BrD} 2$ to $\mathrm{BrD} 4$ with 


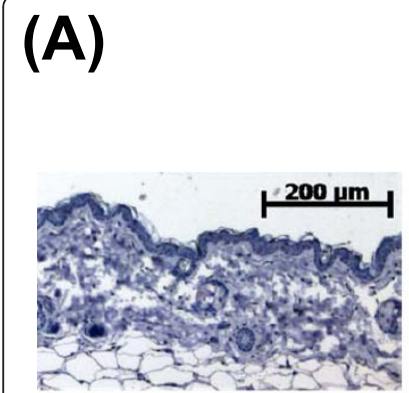

Untreated

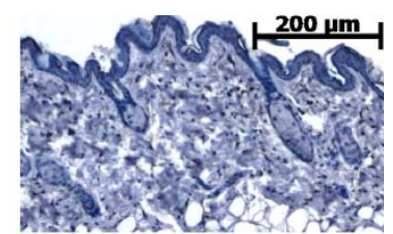

Acetone

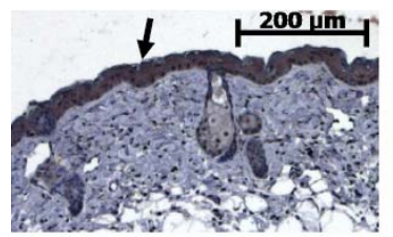

TPA

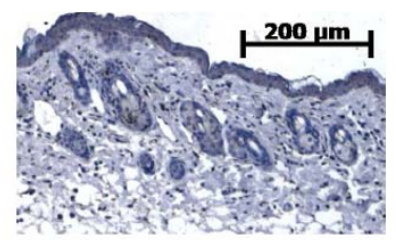

TPA + BrD1 (1mg/site)

\section{(B)}

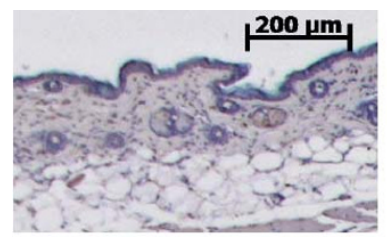

Untreated

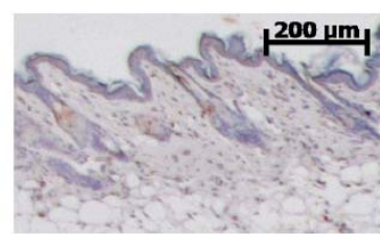

Acetone

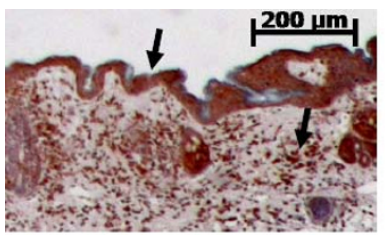

TPA

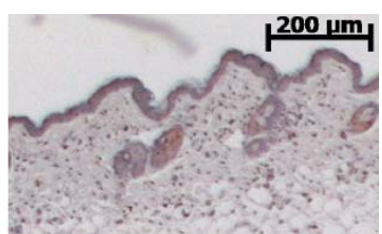

TPA + BrD1 (1mg/site)

Figure 3 BrD1 suppresses TPA-induced iNOS expression in mouse skin. Abdominal skins of female C57BL/6 mice were topically treated as described in Figure 1. Longitudinal tissue sections of abdominal skins were immunostained for COX-2 (A) and iNOS (B) proteins and counterstained with hematoxylin, as described in Materials and Methods. Positive staining for COX-2 and iNOS are visualized as brownish cells in the dermis and epidermis (arrow). Data are representative of two independent experiments.

longer ester chains gradually decreased the level of inhibition of IL- 6 expression by these BrDs, suggesting that the steric hindrance of longer acyloxyl groups can inhibit the capacity of the briarane-type diterpenes to inhibit of LPS-induced IL-6 expression (Table 1 and Figure 7A).

\section{Steric hindrance of 12-acyloxyl substituents reduces the} inhibitory bioactivity of briarane-type diterpenes

Our results suggest that steric hindrance of the 12-acyloxyl substituents of BrDs decreases their inhibitory activity. In order to confirm this SAR, we semi-synthesized several analogues of $\mathrm{BrD} 1$ by replacing the hydroxyl group at C-12 with longer acyloxyl groups $\left(\mathrm{C}_{5}, \mathrm{C}_{6}\right.$, $\mathrm{C}_{7}$ and $\mathrm{C}_{10}$ ) (Figure 7B). We then examined the inhibitory effect of BrD1 and these BrD1 analogues (BrD1-5C, BrD1-6C, BrD1-7C and BrD1-10C) on cytokine secretion activity in LPS-stimulated mature DCs. BrD1 significantly inhibited the expression of cytokine IL-6 in LPSstimulated, mature DCs (Figure 7C). This inhibitory activity gradually decreased when the hydroxyl group at
C-12 was replaced by longer acyloxyl groups $\left(\mathrm{C}_{5}\right.$ to $\mathrm{C}_{10}$ ), confirming that steric hindrance of 12-acyloxyl substituents can effectively suppress the capacity of BrDs to inhibit LPS-induced IL-6 expression. These results thus strongly suggest that the 8,17 -epoxide and 12-hydroxyl groups of BrD1 are instrumental in conferring the BrD-mediated inhibitory activity in mouse bone marrow-derived dendritic cells.

\section{Discussion}

The immune response in skin tissues is systemic and sophisticated. A variety of vaccination strategies are administered through the skin to produce antigen-specific immune responses and protect the host [22-25]. The skin is also a useful organ for drug delivery, for example, certain pharmaceuticals can be delivered via the use of a transdermal patch. On the other hand, excessive inflammation or inadequate immune response in skin can lead to various cutaneous diseases such as allergic reactions, psoriasis and even skin cancers. Our laboratories have 


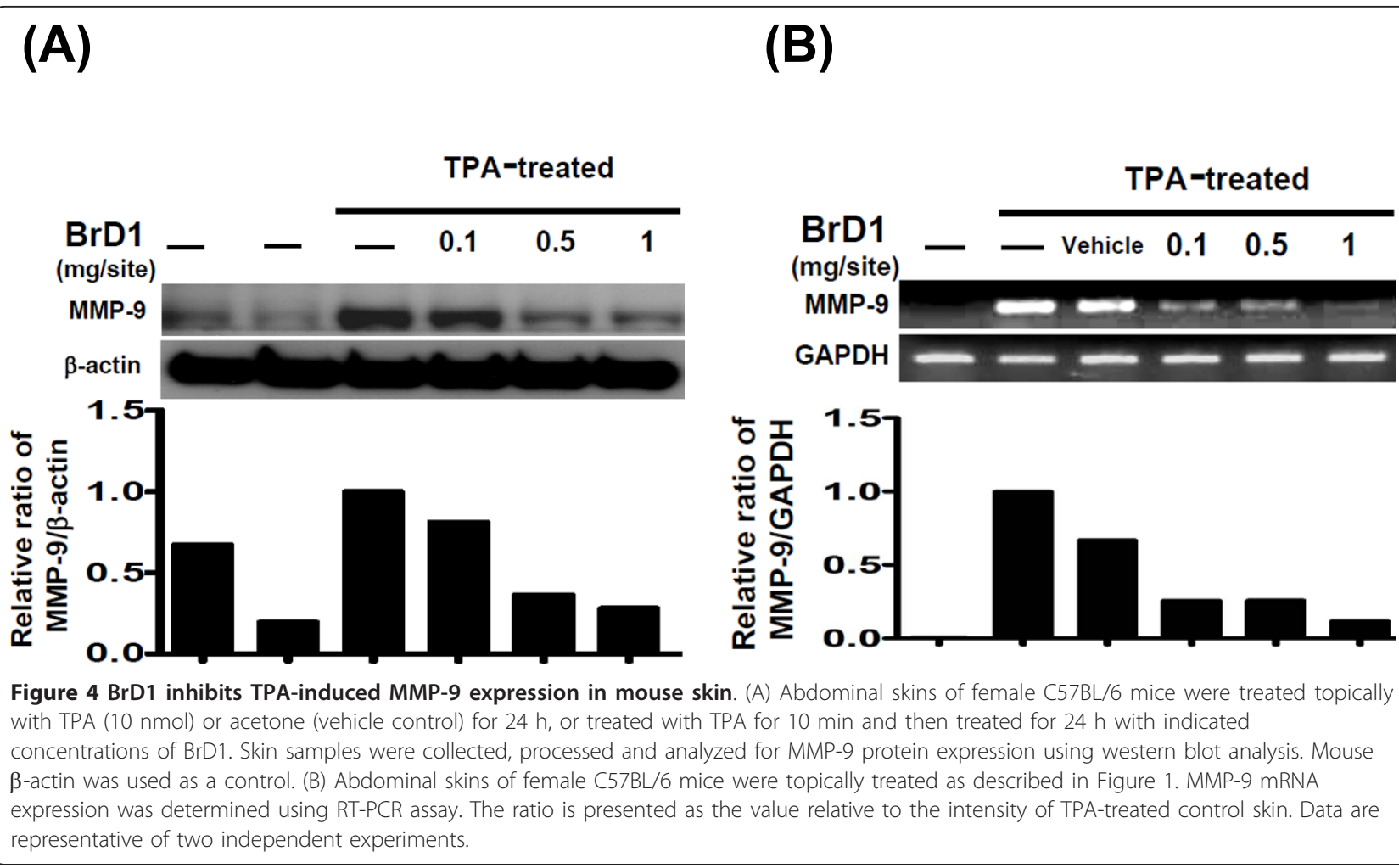

previously demonstrated that a number of phytochemicals derived from medicinal plants or fruits exhibit strong anti-inflammatory effects either on TPA-induced inflammatory mediators or UVB radiation-induced cytokine and MMP expression in mouse skin tissues $[7,11,26]$. In the present study, we showed that a group of marine briarane-type diterpenes, particularly BrD1, can strongly suppress TPA-induced inflammation and dermatitis in a mouse skin model. In combination with our previous studies $[7,10,11,26]$, the current study leads us to suggest that a specific combination of several skin model/system test sets [27] may warrant a systematic evaluation for use as the first in vivo platform for drug screening or efficacy verifications.

Epidermis tissue contains keratinocytes, Langerhans cells and other cells types. When the epidermis is exposed to 12-O-tetradecanoylphorbol-13-acetate (TPA), keratinocytes and Langerhans cells are stimulated through activation of protein kinase $C$ (PKC) resulting in activation of PI3K/Akt, Erk and NF- $\kappa \mathrm{B}$ signaling transduction pathway [28-30]. This activation of Akt and NF- $\kappa$ B activity via TPA stimulation was faithfully observed in the present investigation (Figure 5A), providing a solid baseline from which to investigate the effect of BrD1. Akt and NF- $\kappa$ B play crucial roles in the activation of key inflammatory enzymes such as inducible nitric oxide synthase (iNOS) and cyclooxygenase-2 (COX-2) [31-33]. Interestingly, our results showed that the expression of COX-2 and iNOS was detected mainly in the epidermis after TPA stimulation, indicating that COX-2 and iNOS were mainly released from epidermal cells (likely mainly from keratinocytes) (Figure 3A and 3B). In addition, we also observed considerable sporadic expression of iNOS in the dermal layer after TPA treatment, and BrD1 strongly inhibited this iNOS expression in both the epidermal and dermal layers (Figure 3B). The specific cell types in these two tissue layers that are responsive to $\mathrm{BrD} 1$ inhibition effect need to be further investigated. The major dermis cell types are fibroblasts, macrophages and fabric basophils (mast cells). During inflammation, immune cells, mainly neutrophils, can effectively infiltrate into the dermal layer. Keratinocytes, mast cells and infiltrated neutrophils in skin have been reported to secrete MMP-9 for tissue remodeling in response to inflammation [34-36]. MMP-9 has further been reported to be directly mechanistically involved in the increases of vascular permeability [37-39]. Our results (Figure $4 \mathrm{~A}$ and $4 \mathrm{~B}$ ) show that BrD1 can effectively inhibit TPA-induced MMP-9 expression. We suggest that this may be partly due to the inhibitory effect of $\mathrm{BrD} 1$ on vascular permeability and edema. Together, our present results suggest that BrD1 may suppress the inflammatory responses mediated by different types of cells present in different tissue layers of the skin. Our data (Figure 5A and 5B) also indicate that the molecular mechanisms responsible for the anti-inflammatory 


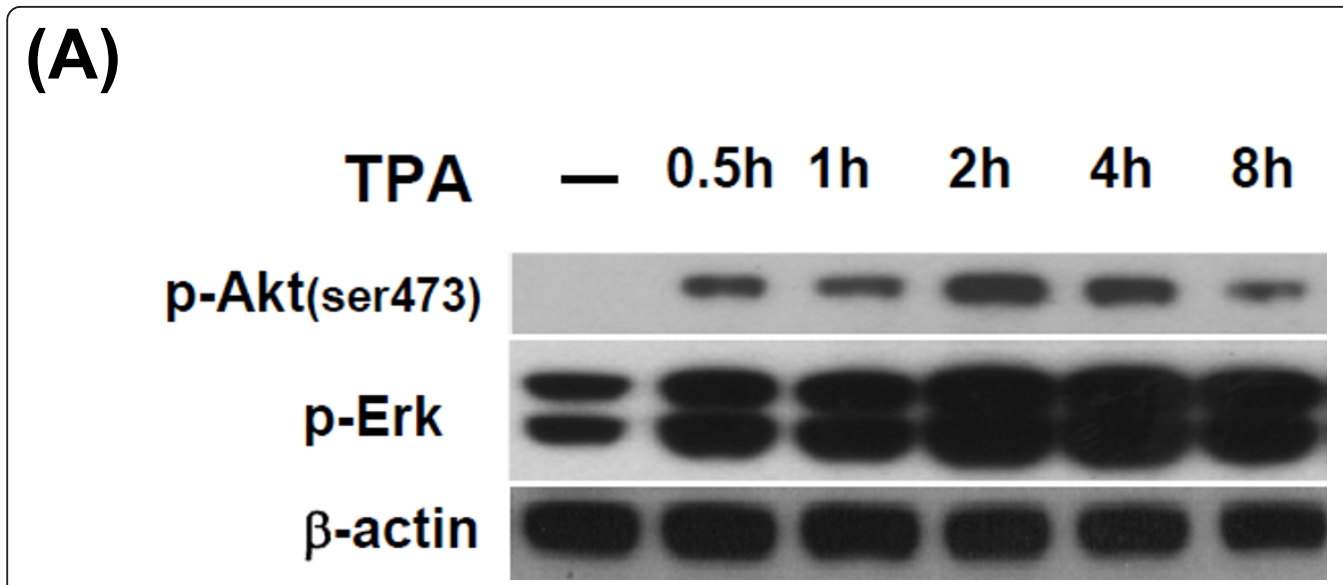

(B)

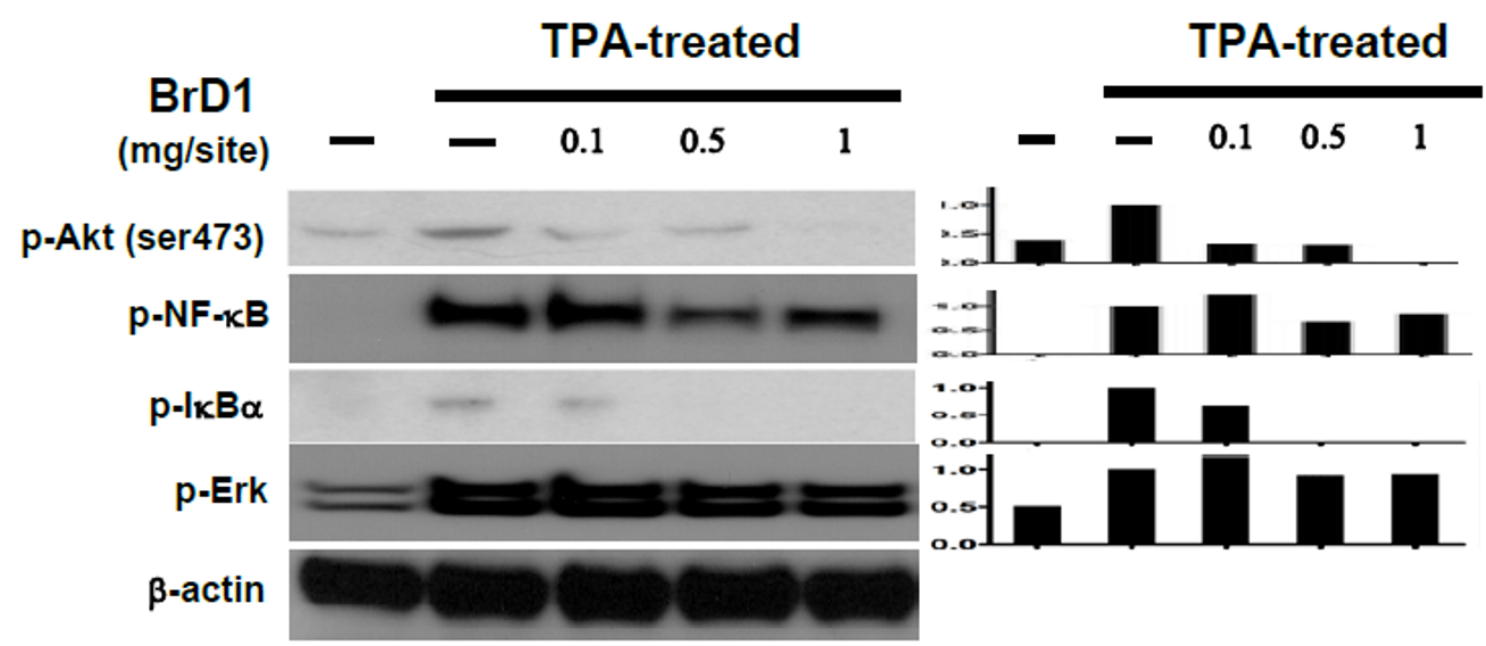

Figure 5 BrD1 inhibits TPA-induced NF-kB and Akt activation in mouse skin. (A) Mouse skin was untreated or TPA-treated for indicated time points, and test skin samples were collected and analyzed for phosphorylation levels of Akt and Erk1/2. (B) Abdominal skins of female C57BL/6 mice were treated topically with TPA $(10 \mathrm{nmol})$ or acetone for $2 \mathrm{~h}$ or treated with TPA for $10 \mathrm{~min}$ and then treated for $2 \mathrm{~h}$ with the indicated concentrations of BrD1 for $2 \mathrm{~h}$. Skin samples were collected, processed and analyzed for the phosphorylation levels of Akt, NF- $\kappa \mathrm{B}$, I $\kappa \mathrm{B} \alpha$ and Erk1/2 by Western blot analysis. Mouse $\beta$-actin was used as a control. The ratio is presented as the value relative to the intensity of TPA-

treated control skin. Data are representative of two independent experiments.

effects of BrD1 may involve the Akt and NF- $\kappa \mathrm{B}$ signaling transduction pathway.

The pro-inflammatory cytokines TNF- $\alpha$ and IL-6, released by dendritic cells, are key mediators of inflammatory and immune responses. It is known that expression of these cytokines is controlled by TLR4-stimulated activation of NF- $\kappa \mathrm{B}$ via regulation of the transcriptional activities of these cytokines in monocytes and dendritic cells. The results from this study show that BrD1 can inhibit the activation of NF- $\kappa \mathrm{B}$ in TPA-induced mouse dermatitis (Figure 5B) and in LPS-stimulated mouse
BMDCs (data not shown). However, BrD1 exhibited a differential inhibitory effect on TNF- $\alpha$ and IL-6 in LPSinduced mouse BMDCs. As seen in Figure 6B, BrD1 strongly inhibited LPS-induced IL-6 expression but only partially inhibited LPS-induced TNF- $\alpha$ expression. In order to distinguish between TNF- $\alpha$ and IL- 6 inhibition, a time course study of LPS-induced TNF- $\alpha$ and IL- 6 expression was performed. After LPS stimulation, a relatively high level of expression of TNF- $\alpha$ was detected between $1 \mathrm{~h}$ and $2 \mathrm{~h}$ post-treatment, and an elevated level of IL- 6 expression was detected between $2 \mathrm{~h}$ and 4 


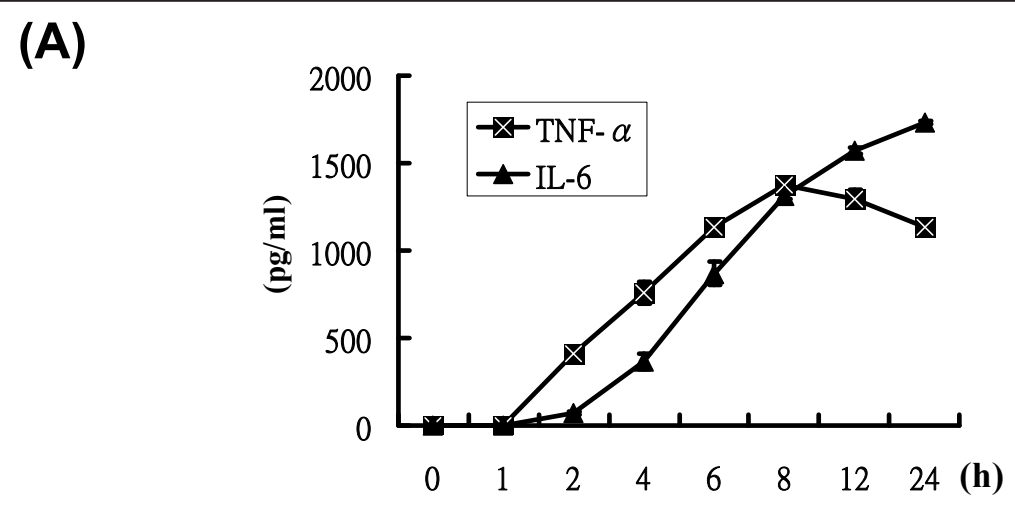

(B)
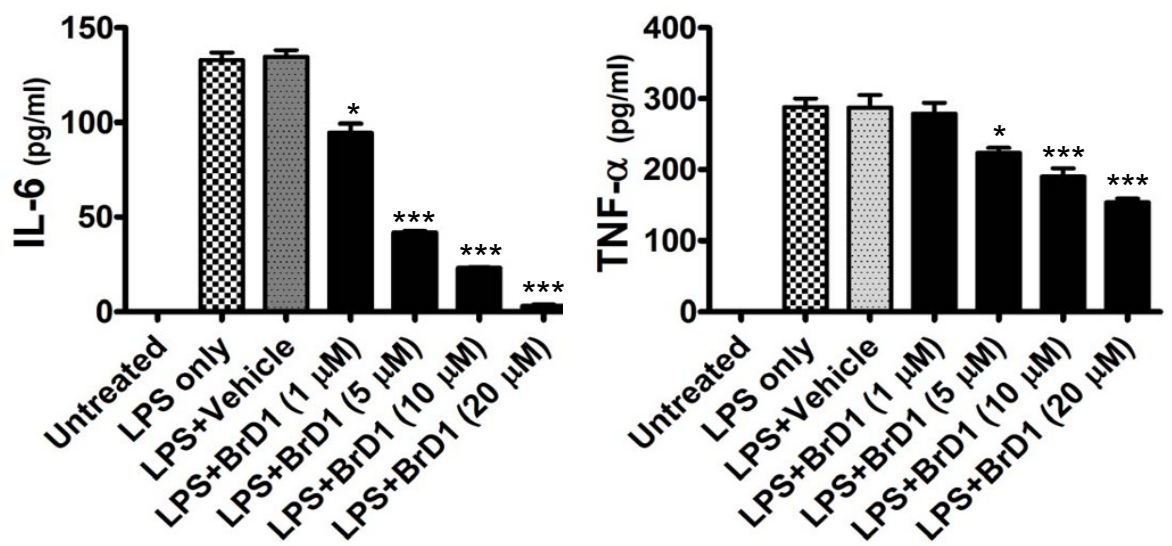

Figure 6 BrD1 inhibits LPS-induced IL- 6 and TNF- $\alpha$ expression in mouse BMDCs. (A) BMDCs from C57BL/6 mice were treated with LPS (100 ng/ml) for 1 to $24 \mathrm{~h}$. (B) BMDCs from C57BL/6 mice were treated with LPS (100 ng/ml) for $24 \mathrm{~h}$ or LPS plus BrD1 at different concentrations for $24 \mathrm{~h}$. Levels of IL-6 and TNF- $\alpha$ proteins in supernatants of conditioned media were analyzed by ELISA. ${ }^{*}, P<0.05$, and ${ }^{* * *}, P<$ 0.001 versus LPS control. Data are representative of two independent experiments.

h post-treatment (Figure 6A). Our previous findings and those of others have shown evidence of post-transcriptional regulation of TNF- $\alpha$ pre-mRNA in resting Tcells, B-cells and monocytes [40-42]. LPS-induced mRNA splicing can lead to massive and rapid expression of TNF- $\alpha$ mRNA in monocytes [42]. The differential effect on expression of TNF- $\alpha$ and IL- 6 in LPSactivated BMDCs seen in this study may have implications for the future use of BrD1 in pharmaceutical applications. The detailed molecular mechanisms responsible for this differential effect warrant further investigation.

\section{Conclusions}

In summary, we conclude that marine briarane-type diterpene (BrD1) can not only effectively suppress TPA-induced vascular permeability and edema, but can also decrease the expression of COX-2, iNOS and MMP-9, and reduce the activation of NF- $\kappa$ B and Akt in test mouse skin tissues. In addition, BrD1 can also strongly inhibit IL-6 expression in LPS-stimulated BMDCs, a key immunoregulatory cell type. The 8, 17-
Table 1 Inhibitory effect of briarane-type diterpenes from marine coral Briareum excavatumon on LPS-induced IL-6 in mouse BMDCs

\begin{tabular}{|c|c|c|}
\hline \multicolumn{3}{|c|}{ IL-6 (\% of LPS control) } \\
\hline Code & Name $(20 \mu \mathrm{M})$ & $\%($ Mean \pm SD $)$ \\
\hline BrD1 & $\begin{array}{c}\text { Excavatolide } \\
\text { B }\end{array}$ & $2.4 \pm 0.93$ \\
\hline $\mathrm{BrD2}$ & $\begin{array}{c}\text { Excavatolide } \\
\mathrm{K}\end{array}$ & $22.5 \pm 4.5$ \\
\hline $\mathrm{BrD3}$ & $\begin{array}{c}\text { Excavatolide } \\
\text { F }\end{array}$ & $26.2 \pm 4.0$ \\
\hline BrD4 & $\begin{array}{c}\text { Briaexcavatolide } \\
\text { R }\end{array}$ & $34.7 \pm 8.6$ \\
\hline BrD5 & $\begin{array}{c}\text { Excavatolide } \\
Z\end{array}$ & $37.0 \pm 9.6$ \\
\hline $\mathrm{BrD6}$ & $\begin{array}{c}\text { Briaexcavatolide } \\
\text { B }\end{array}$ & $56.6 \pm 2.1$ \\
\hline BrD7 & $\begin{array}{c}\text { Briaexcavatolide } \\
\text { K }\end{array}$ & $61.3 \pm 9.5$ \\
\hline BrD8 & $\begin{array}{c}\text { Briaexcavatolide } \\
\mathrm{H}\end{array}$ & $81.3 \pm 1.9$ \\
\hline
\end{tabular}

IL-6 in supernatants of diterpenes BrD1 to BrD8 were determined by using a standard sandwich ELISA. Results are expressed as a percentage of LPSinduced IL-6 expression. 


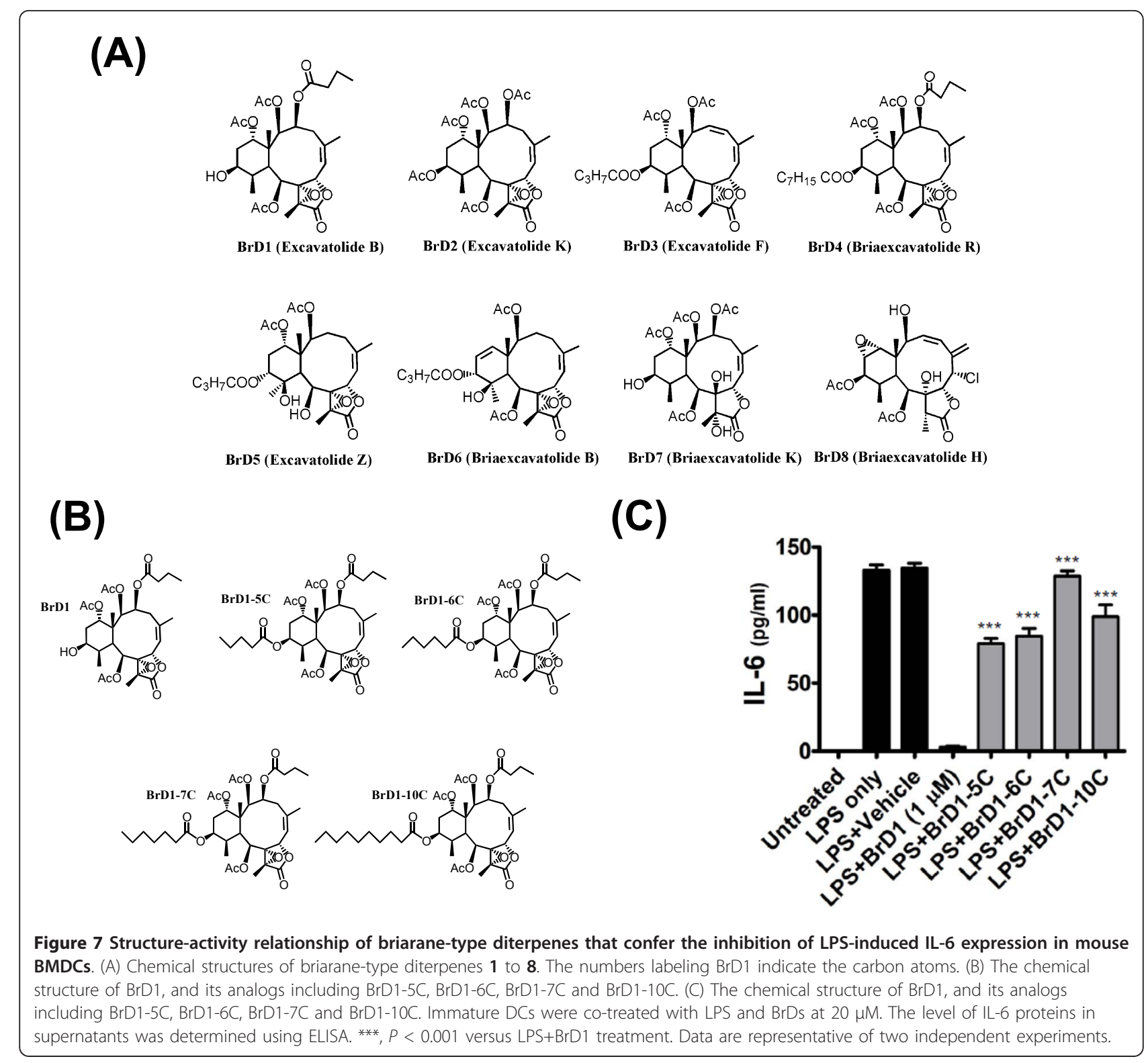

epoxide of the BrDs apparently contribute stereochemically to the inhibition of IL- 6 expression, and steric hindrance of the 12-acyloxyl substituents was found to effectively reduce the inhibitory bioactivity of $\mathrm{BrD} 1$. In view of the unique structure and specific active functional group of BrD1, we suggest this marine natural product should be further evaluated for development as an immunotherapeutic agent for control of inflammation and skin diseases, and for other healthcare applications.

\section{Acknowledgements}

We thank Dr. Heiko Kuhn of the Academia Sinica Institute of Molecular Biology and Agricultural Biotechnology Research Center Joint Editors' Office, and Ms. Ruth Giordano of Dr. Ning-Sun Yang's laboratory for editing the manuscript. We also thank Dr. Chia-Chung Hou of the Academia Sinica Agricultural Biotechnology Research Center and Ms. Hui-Ming Chen of the Academia Sinica Agricultural Biotechnology Research Center for valuable suggestions and thank Ms. Chia-Chi Wu of the Academia Sinica Agricultural Biotechnology Research Center and Ms. Li-Ting Huang of the Academia Sinica Agricultural Biotechnology Research Center for performing some tests. This work was supported by a grant (NSC98-2113-M-110-002-MY3) from the National Science Council, Executive Yuan, Taiwan, ROC; and by grants (CCMP 99-RD-067 and CCMP100-RD-019) from the Committee on Chinese Medicine and Pharmacy, Department of Health, Executive Yuan, Taiwan, ROC

\section{Author details}

Agricultural Biotechnology Research Center, Academia Sinica, Taiwan. ${ }^{2}$ Department of Marine Biotechnology and Resources, National Sun Yat-sen University, Kaohsiung, Taiwan. ${ }^{3}$ Graduate Institute of Life Science, National Defense Medical Center, Taipei, Taiwan. ${ }^{4}$ Department of Life Science, National Central University, Taoyuan County, Taiwan. ${ }^{5}$ Department of Life Science, National Taiwan University, Taipei, Taiwan. ${ }^{6}$ Asia-Pacific Ocean Research Center, National Sun Yat-Sen University, Kaohsiung, Taiwan. 


\section{Authors' contributions}

Wen-Chi Wei initiated the study, designed and performed the experiments, analyzed data, and wrote the manuscript; Sheng-Yen Lin and Yi-Jyun Chen designed the research, analyzed data, and wrote the manuscript; Chih-Chun Wen and Arulselvan Palanisamy performed some tests and analyzed data; Chiung-Yao Huang contributed vital new reagents; Ning-Sun Yang and JyhHorng Sheu established strategies and approaches, designed the research and wrote the manuscript. All authors read and approved the final manuscript.

\section{Competing interests}

The authors declare that they have no competing interests.

Received: 17 November 2011 Accepted: 21 December 2011 Published: 21 December 2011

\section{References}

1. Kupper TS, Fuhlbrigge RC: Immune surveillance in the skin: mechanisms and clinical consequences. Nature reviews Immunology 2004, 4:211-222.

2. Nestle FO, Di Meglio P, Qin JZ, Nickoloff BJ: Skin immune sentinels in health and disease. Nature reviews Immunology 2009, 9:679-691.

3. Jermy A: Innate immunity: Bacteria ensure injury is only skin deep. Nature Reviews Immunology 2010, 10:1.

4. Nagaoka I, Hirota S: Increased expression of matrix metalloproteinase-9 in neutrophils in glycogen-induced peritoneal inflammation of guinea pigs. Inflamm Res 2000, 49:55-62.

5. De Vry CG, Valdez M, Lazarov M, Muhr E, Buelow R, Fong T, lyer S: Topical application of a novel immunomodulatory peptide, RDP58, reduces skin inflammation in the phorbol ester-induced dermatitis model. J Invest Dermatol 2005, 125:473-481.

6. Cumberbatch M, Dearman RJ, Kimber I: Constitutive and inducible expression of interleukin- 6 by Langerhans cells and lymph node dendritic cells. Immunology 1996, 87:513-518.

7. Stanifortha V, Huang WC, Aravindaram K, Yang NS: Ferulic acid, a phenolic phytochemical, inhibits UVB-induced matrix metalloproteinases in mouse skin via posttranslational mechanisms. The Journal of Nutritional Biochemistry.

8. Nair HB, Sung B, Yadav VR, Kannappan R, Chaturvedi MM, Aggarwal BB: Delivery of antiinflammatory nutraceuticals by nanoparticles for the prevention and treatment of cancer. Biochem Pharmacol 2010, 80:1833-1843

9. Wang CY, Staniforth V, Chiao MT, Hou CC, Wu HM, Yeh KC, Chen CH, Hwang Pl, Wen TN, Shyur LF, Yang NS: Genomics and proteomics of immune modulatory effects of a butanol fraction of echinacea purpurea in human dendritic cells. BMC Genomics 2008, 9:479.

10. Staniforth V, Wang SY, Shyur LF, Yang NS: Shikonins, phytocompounds from Lithospermum erythrorhizon, inhibit the transcriptional activation of human tumor necrosis factor alpha promoter in vivo. J Biol Chem 2004, 279:5877-5885

11. Staniforth V, Chiu LT, Yang NS: Caffeic acid suppresses UVB radiationinduced expression of interleukin-10 and activation of mitogenactivated protein kinases in mouse. Carcinogenesis 2006, 27:1803-1811.

12. Marris E: Marine natural products: drugs from the deep. Nature 2006, 443:904-905.

13. Blunt JW, Copp BR, Munro MH, Northcote PT, Prinsep MR: Marine natural products. Nat Prod Rep 2005, 22:15-61.

14. Sheu JH, Sung PJ, Cheng MC, Liu HY, Fang LS, Duh CY, Chiang MY: Novel cytotoxic diterpenes, excavatolides A-E, isolated from the Formosan gorgonian Briareum excavatum. J Nat Prod 1998, 61:602-608.

15. Sheu JH, Sung PJ, Su JH, Wang GH, Duh CY, Shen YC, Chiang MY, Chen IT: Excavatolides U-Z, new briarane diterpenes from the gorgonian briareum excavatum. J Nat Prod 1999, 62:1415-1420.

16. Thurston G, Suri C, Smith K, McClain J, Sato TN, Yancopoulos GD, McDonald DM: Leakage-resistant blood vessels in mice transgenically overexpressing angiopoietin-1. Science 1999, 286:2511-2514.

17. Yin SY, Wang WH, Wang BX, Aravindaram K, Hwang PI, Wu HM, Yang NS: Stimulatory effect of Echinacea purpurea extract on the trafficking activity of mouse dendritic cells: revealed by genomic and proteomic analyses. BMC genomics 2010, 11:612.
18. Rao TS, Currie JL, Shaffer AF, Isakson PC: Comparative evaluation of arachidonic acid (AA)- and tetradecanoylphorbol acetate (TPA)-induced dermal inflammation. Inflammation 1993, 17:723-741.

19. Vane JR, Mitchell JA, Appleton I, Tomlinson A, Bishop-Bailey D, Croxtall J, Willoughby DA: Inducible isoforms of cyclooxygenase and nitric-oxide synthase in inflammation. Proc Natl Acad Sci USA 1994, 91:2046-2050.

20. Stamenkovic I: Extracellular matrix remodelling: the role of matrix metalloproteinases. J Pathol 2003, 200:448-464.

21. Shakarijan MP, Bhatt P, Gordon MK, Chang YC, Casbohm SL, Rudge TL, Kiser RC, Sabourin CL, Casillas RP, Ohman-Strickland P, Riley DJ, Gerecke DR: Preferential expression of matrix metalloproteinase- 9 in mouse skin after sulfur mustard exposure. J Appl Toxicol 2006, 26:239-246.

22. Romani N, Thurnher M, Idoyaga J, Steinman RM, Flacher V: Targeting of antigens to skin dendritic cells: possibilities to enhance vaccine efficacy. Immunol Cell Biol 2010, 88:424-430.

23. Rakhmilevich AL, Turner J, Ford MJ, McCabe D, Sun WH, Sondel PM, Grota K, Yang NS: Gene gun-mediated skin transfection with interleukin 12 gene results in regression of established primary and metastatic murine tumors. Proceedings of the National Academy of Sciences of the United States of America 1996, 93:6291-6296.

24. Oshikawa K, Shi F, Rakhmilevich AL, Sondel PM, Mahvi DM, Yang NS: Synergistic inhibition of tumor growth in a murine mammary adenocarcinoma model by combinational gene therapy using IL-12, proIL-18, and IL-1 beta converting enzyme CDNA. Proceedings of the National Academy of Sciences of the United States of America 1999, 96:13351-13356.

25. Aravindaram $\mathrm{K}, \mathrm{Yu} \mathrm{HH}$, Lan CW, Wang PH, Chen YH, Chen HM, Yagita H, Yang NS: Transgenic expression of human gp100 and RANTES at specific time points for suppression of melanoma. Gene Ther 2009, 16:1329-1339.

26. Chiang YM, Lo CP, Chen YP, Wang SY, Yang NS, Kuo YH, Shyur LF: Ethyl caffeate suppresses NF-kappaB activation and its downstream inflammatory mediators, iNOS, COX-2, and PGE2 in vitro or in mouse skin. Br J Pharmacol 2005, 146:352-363.

27. Wang JH, Lin KF, Benson SA, Sun SJ, Cheng WM, Wang SY, Shyur LF, Yang NS: Tissue Array Transgene Expression System for the Evaluation of Effect of Medicinal Herbs on Wound-healing. Journal of Genetics and Molecular Biology 2003, 14:133-144.

28. Ridd K, Dhir S, Smith AG, Gant TW: Defective TPA signalling compromises HaCat cells as a human in vitro skin carcinogenesis model. Toxicol In Vitro 2010, 24:910-915.

29. Van Dross RT, Hong X, Pelling JC: Inhibition of TPA-induced cyclooxygenase-2 (COX-2) expression by apigenin through downregulation of Akt signal transduction in human keratinocytes. Mol Carcinog 2005, 44:83-91.

30. Sur I, Ulvmar M, Jungedal R, Toftgard R: Inhibition of NF-kappaB signaling interferes with phorbol ester-induced growth arrest of keratinocytes in a TNFR1-independent manner. J Recept Signal Transduct Res 2009, 29:44-51.

31. Bayon Y, Ortiz MA, Lopez-Hernandez FJ, Gao F, Karin M, Pfahl M, Piedrafita FJ: Inhibition of IkappaB kinase by a new class of retinoidrelated anticancer agents that induce apoptosis. Mol Cell Biol 2003, 23:1061-1074.

32. ChiU FL, Lin JK: Tomatidine inhibits iNOS and COX-2 through suppression of NF-kappaB and JNK pathways in LPS-stimulated mouse macrophages. FEBS Lett 2008, 582:2407-2412.

33. Jang BC, Paik JH, Kim SP, Shin DH, Song DK, Park JG, Suh MH, Park JW, Suh SI: Catalase induced expression of inflammatory mediators via activation of NF-kappaB, PI3K/AKT, p70S6K, and JNKs in BV2 microglia. Cell Signal 2005, 17:625-633.

34. Harvima IT: Induction of matrix metalloproteinase-9 in keratinocytes by histamine. J Invest Dermatol 2008, 128:2748-2750.

35. Kahari VM, Saarialho-Kere U: Matrix metalloproteinases in skin. Exp Dermatol 1997, 6:199-213.

36. Rijken F, Bruijnzeel PL, van Weelden H, Kiekens RC: Responses of black and white skin to solar-simulating radiation: differences in DNA photodamage, infiltrating neutrophils, proteolytic enzymes induced, keratinocyte activation, and IL-10 expression. J Invest Dermatol 2004, 122:1448-1455.

37. Lee S, Jilani SM, Nikolova GV, Carpizo D, Iruela-Arispe ML: Processing of VEGF-A by matrix metalloproteinases regulates bioavailability and vascular patterning in tumors. J Cell Biol 2005, 169:681-691. 
38. Raffetto JD, Khalil RA: Matrix metalloproteinases and their inhibitors in vascular remodeling and vascular disease. Biochem Pharmacol 2008, 75:346-359.

39. Wu J, Akaike T, Hayashida K, Okamoto T, Okuyama A, Maeda H: Enhanced vascular permeability in solid tumor involving peroxynitrite and matrix metalloproteinases. Jpn J Cancer Res 2001, 92:439-451.

40. Yang Y, Chang JF, Parnes JR, Fathman CG: T cell receptor (TCR) engagement leads to activation-induced splicing of tumor necrosis factor (TNF) nuclear pre-mRNA. J Exp Med 1998, 188:247-254.

41. Li YY, Yang Y, Bao M, Edwards CK, Parnes JR: Mouse splenic B lymphocyte activation using different activation stimuli induces in vitro splicing of tumor necrosis factor-alpha nuclear pre-mRNA. Mol Immunol 2006, 43:613-622.

42. Chiu SC, Yang NS: Inhibition of tumor necrosis factor-alpha through selective blockade of Pre-mRNA splicing by shikonin. Molecular pharmacology 2007, 71:1640-1645.

doi:10.1186/1423-0127-18-94

Cite this article as: Wei et al:: Topical application of marine briaranetype diterpenes effectively inhibits 12-O-tetradecanoylphorbol-13acetate-induced inflammation and dermatitis in murine skin. Journal of Biomedical Science 2011 18:94.

\section{Submit your next manuscript to BioMed Central} and take full advantage of:

- Convenient online submission

- Thorough peer review

- No space constraints or color figure charges

- Immediate publication on acceptance

- Inclusion in PubMed, CAS, Scopus and Google Scholar

- Research which is freely available for redistribution

Submit your manuscript at www.biomedcentral.com/submit
() Biomed Central 